\title{
Belief base merging as a game
}

\author{
Sébastien Konieczny \\ CRIL - CNRS \\ Université d'Artois \\ 62300 Lens (France) \\ konieczny@cril.univ-artois.fr
}

ABSTRACT. We propose in this paper a new family of belief merging operators, that is based on a game between sources : until a coherent set of sources is reached, at each round a contest is organized to find out the weakest sources, then those sources has to concede (weaken their point of view). This idea leads to numerous new interesting operators (depending of the exact meaning of "weakest" and "concede", that gives the two parameters for this family) and opens new perspectives for belief merging. Some existing operators are also recovered as particular cases. Those operators can be seen as a special case of Booth's Belief Negotiation Models [BOO 02], but the achieved restriction forms a consistent family of merging operators that worths to be studied on its own.

KEYWORDS: belief merging, belief negotiation.

\section{Introduction}

The problem of (propositional) belief merging [REV 97, LIN 99, LIB 98, KON 99, KON 02a, KON 04] can be summarized by the following question: given a set of sources (propositional belief bases) that are (typically) mutually inconsistent, how do we obtain a coherent belief base reflecting the beliefs of the set?

The idea here is that some/each sources has to concede on some points in order to solve the conflicts. If one has some notion of relative reliability between sources, it is enough and sensible to force the less reliable ones to give up first. There is a variety of different means to do that, which has provided a large literature, e.g. [CHO 93, CHO 95, CHO 98, BEN 98a, BEN 98b]. But often we do not have such information, and even if we get it, it remains the more fundamental problem of how to merge sources of equal reliability [KON 99, KON 02a].

In this paper we will investigate the merging methods based on a notion of game between the sources. The intuitive idea is simple: when trying to impose its wish, each source will try to form some coalition with the closest (more compatible) other 
sources. So the source that is the "furthest" from the other ones will certainly be the weakest one. And it will be that source that will have to concede first. In this work, we will not focus on how the coalitions form, we only take this idea to designate the weakest ones.

So the merging is based on the following game: until a coherent set of sources is reached, at each round a contest is organized to find out the weakest sources, then those sources have to concede (weaken their point of view).

We can state several intuitions and justifications for the use of such operators. We have already given the first one: coalition with near-minded sources. In a group decision process between rational sources, it can be sensible to expect the sources to look for near-minded sources in order to find help to defend their view, so the "furthest" source is the more likely to have to concede on its view.

A second intuition is the one given by a social pressure on the sources. When confronting several points of view, usually people that have the more exotic views try to change their opinion in order to be accepted by the other members of the group, so opinions that are defended by the least number of sources are usually given up more easily in the negotiation process.

A last intuition that gives the main rationale for that kind of operator is Condorcet's Jury theorem. This theorem states that if all the members of a jury are reliable (in the sense that they have greater than $50 \%$ chance to find the truth), then listening to the majority is the more rational choice.

After stating some useful definitions and notations in Section 2, we will define the new family of operators we propose in Section 3. The definition will use a notion of weakening and choice functions. We will explore these notions in Section 4. We will give some examples of specific operators in Section 5 in order to illustrate their behaviour. We will look at the logical properties of those operators in Section 6. In Section 7 we will look at the links between this work and related works (especially Booth's proposal [BOO 01, BOO 02]). We conclude in Section 8 with some open issues and perspectives of this work.

\section{Definitions}

We consider a propositional language $\mathcal{L}$ over a finite alphabet $\mathcal{P}$ of propositional symbols. An interpretation is a function from $\mathcal{P}$ to $\{0,1\}$. The set of all the interpretations is denoted $\mathcal{W}$. An interpretation $\omega$ is a model of a formula $\varphi$, noted $\omega \models \varphi$, if and only if it makes it true in the usual classical truth functional way. Let $\varphi$ be a formula, $\bmod (\varphi)$ denotes the set of models of $\varphi$, i.e. $\bmod (\varphi)=\{\omega \in \mathcal{W} \mid \omega \models \varphi\}$. Conversely, let $X$ be a set of interpretations, $\operatorname{form}(X)$ denotes the formula (up to logical equivalence) whose set of models is $X$. 
A belief base $\varphi$ is a consistent propositional formula (or, equivalently, a finite consistent set of propositional formulae considered conjunctively). Let us note $\mathcal{K}$ the set of all belief bases.

Let $\varphi_{1}, \ldots, \varphi_{n}$ be $n$ belief bases (not necessarily different). We call belief profile the multi-set $\Psi$ consisting of those $n$ belief bases: $\Psi=\left(\varphi_{1}, \ldots, \varphi_{n}\right)$ (i.e. two sources can have the same belief base). We note $\bigwedge \Psi$ the conjunction of the belief bases of $\Psi$, i.e. $\wedge \Psi=\varphi_{1} \wedge \cdots \wedge \varphi_{n}$. We say that a belief profile is consistent if $\bigwedge \Psi$ is consistent. The multi-set union will be noted $\sqcup$ and the multi-set inclusion will be noted $\sqsubseteq$. The cardinal of a finite (multi-)set $\Psi$ is noted \# $\Psi$ ) (the cardinal of a finite multi-set is the sum of the numbers of occurrences of each of its elements).

Let us define those multi-set notions more formally. As a set $\Phi$ (from elements in a set A) can be defined from its characteristic function $\chi_{\Phi}: A \rightarrow\{0,1\}$, a multiset $\Psi$ (from elements in a set A) can be defined from its characteristic function $\chi_{\Psi}$ : $A \rightarrow \mathbb{N}$, where $\mathbb{N}$ is the set of nonnegative integers ${ }^{1}$. Then the multi-set union, noted $\sqcup$, is defined as $\chi_{\Psi \sqcup \Psi^{\prime}}=\chi_{\Psi}+\chi_{\Psi^{\prime}}$. The cardinality of a multi-set is defined as $\#(\Psi)=\sum_{a \in A} \chi_{\Psi}(a)$.

Indeed, all set notions used in this paper (subset, inclusion, union, etc.), are for multi-sets. For the sake of simplicity, and since it cannot lead to confusion since those notions are a generalization of the set ones, we will omit the "multi-".

Let $\mathcal{E}$ be the set of all finite non-empty belief profiles.

Two belief profiles $\Psi_{1}$ and $\Psi_{2}$ are said to be equivalent $\left(\Psi_{1} \equiv \Psi_{2}\right.$ ) if and only if there is a bijection between $\Psi_{1}$ and $\Psi_{2}$ such that each belief base of $\Psi_{1}$ is logically equivalent to its image in $\Psi_{2}$.

\section{Belief game model}

In [BOO 01, BOO 02] Richard Booth proposes a framework for merging sources of information incrementally. He named this framework "Belief Negotiation Model" (BNM). In this work we will use the name "Belief Game Model" (BGM) because in our framework there is no room for negotiation, so we find it more accurate and it allows us to make a distinction in this paper between Booth's proposal and ours. The BGM framework can be seen as a restriction of Booth's BNM framework: the main differences between Booth's proposal and our is that Booth's one take the sources as candidates to weakening, whereas we restrict ourselves to "points of view" (logical content of the sources). That means that in Booth's if one source has to weaken, it can be the case that another source with exactly the same beliefs do not have to weaken too (that is not allowed in our framework). Our proposal involves more anonymity by saying that only beliefs decide who has to weaken, not the identity of one source. Similarly, the choice functions are more "Markovian" in our framework than in Booth's one, that means that we only look at the current profile to choose the bases, whereas in

1. if $a \in A, \chi_{\Psi}(a)=n$ intuitively means that $a$ appears $n$ times in the multi-set $\Psi$. 
Booth's work, one can use the whole history of profiles to make the choice. We think that those hypothesis are more realistic (and necessary) on a belief merging point of view, whereas Booth's framework allows to model more generalized negotiation schemes, where one can decide for example that each source has to weaken one after the other (see Section 7 for a deeper comparison of the two approaches).

DEFINITION 1. - A choice function is a function $g: \mathcal{E} \rightarrow \mathcal{E}$ such that:

$-g(\Psi) \sqsubseteq \Psi$

- If $\bigwedge \Psi \not \equiv \top$, then $\exists \varphi \in g(\Psi)$ s.t. $\varphi \not \equiv \top$

- If $\Psi \equiv \Psi^{\prime}$, then $g(\Psi) \equiv g\left(\Psi^{\prime}\right)$

The choice function aims to find which are the sources that must weaken at a given round (see definition 3). As the weakening function aims to weaken the belief base, and as there is no weaker base than a tautological one, the second condition states that at least one non-tautological base must be selected. So it states that at each round at least one base will be weaken. This condition is necessary to ensures to always reach a result with Belief Game Model. Note that a consequence of this condition is that we have $g(\Psi) \neq \emptyset$ as soon as the profile contains at least one non-tautological base. Last condition is an irrelevance of syntax condition. It states that the selection of the bases to weaken does not depend on the particular form of the bases, but only on their informational content. Note that we also have an additional property: anonymity, that means that the result does not depend on the "name" of the source, but only on its point of view. This is due to the fact that we work with multi-sets, that are equivalent by permutation. If one works with another representation (ordered lists of sources for example), this anonymity property can be given by the last condition, provided that the equivalence between two belief profiles is rightly defined (as in Section 2).

DEFINITION 2. - A weakening function is a function $\mathbf{\nabla}: \mathcal{L} \rightarrow \mathcal{L}$ such that:

$$
\begin{aligned}
& -\varphi \vdash \mathbf{\nabla}(\varphi) \\
& - \text { If } \varphi \equiv \mathbf{\nabla}(\varphi), \text { then } \varphi \equiv \top \\
& - \text { If } \varphi \equiv \varphi^{\prime}, \text { then } \mathbf{\nabla}(\varphi) \equiv \mathbf{\nabla}\left(\varphi^{\prime}\right)
\end{aligned}
$$

The weakening function aims to give the new beliefs of a source that have been chosen to be weakened. The two first conditions ensure that the base will be replaced by a strictly weaker one (unless the base is already a tautological one). The last condition is an irrelevance of syntax requirement : the result of the weakening must only depend on the information conveyed by the base, not on its syntactical form.

We extend the weakening functions on belief profiles as follows: let $\Psi^{\prime}$ be a subset of $\Psi$,

$$
\boldsymbol{\nabla}_{\Psi^{\prime}}(\Psi)=\bigsqcup_{\varphi \in \Psi^{\prime}} \boldsymbol{\nabla}(\varphi) \sqcup \bigsqcup_{\varphi \in \Psi \backslash \Psi^{\prime}} \varphi
$$

This means that we only weaken the belief bases of $\Psi$ that are in $\Psi^{\prime}$, and the other ones do not change. 
Definition 3. - A Belief Game Model is a pair $\mathcal{N}=\langle g, \mathbf{\nabla}\rangle$ where $g$ is a choice function and $\mathbf{\nabla}$ is a weakening function.

The solution to a belief profile $\Psi$ for a Belief Game Model $\mathcal{N}=\langle g, \mathbf{v}\rangle$, noted $\mathcal{N}(\Psi)$, is the belief profile $\Psi_{\mathcal{N}}$, defined as:

$-\Psi_{0}=\Psi$

$-\Psi_{i+1}=\nabla_{g\left(\Psi_{i}\right)}\left(\Psi_{i}\right)$

$-\Psi_{\mathcal{N}}$ is the first $\Psi_{i}$ that is consistent

So the solution to a belief profile is the result of a game on the beliefs of the sources. At each round there is a contest to find out the weakest bases (the losers), and the losers have to concede on their belief by weakening them.

It may prove reasonable that each source has its own weakening function, that denotes different conceding politics. After all, the point is that the source has to weaken its beliefs, not how she does so. So we can figure out a generalization of the belief game model, where there is no one weakening function, but one for each source ${ }^{2}$. But this is not the point in this paper, so we will suppose that there is a unique weakening function for all the sources.

In some cases, the result of the merging has to obey some constraints (physical constraints, norms, etc...). We will assume that these integrity constraints are encoded as a propositional formula (a belief base), and we will note this base $\mu$. Then we introduce the following notion:

DEFINITION 4. - The solution to a belief profile $\Psi$ for a Belief Game Model $\mathcal{N}=$ $\langle g, \mathbf{\nabla}\rangle$ under the integrity constraints $\mu$, noted $\mathcal{N}_{\mu}(\Psi)$, is the belief profile $\Psi_{\mathcal{N}}^{\mu}$ defined as:

$-\Psi_{0}=\Psi$

$-\Psi_{i+1}=\nabla_{g\left(\Psi_{i}\right)}\left(\Psi_{i}\right)$

$-\Psi_{\mathcal{N}}^{\mu}$ is the first $\Psi_{i}$ that is consistent with $\mu$

Often in the following in this paper we will call result of the merging operator $(\mathrm{Be}-$ lief Game Model), the belief base $\bigwedge \Psi_{\mathcal{N}}^{\mu} \wedge \mu$. This abuse of notation is not problematic, since this belief base denotes the consensus point obtained by the belief profile $\Psi_{\mathcal{N}}^{\mu}$ solution of the Belief Game Model process.

Note that the definition of the Belief Game Model and of the weakening and choice functions ensures that each belief profile $\Psi$ has a solution as soon as the constraints $\mu$ are consistent.

2. Technically, it forces to drop out the weakening function from the belief game model $\mathcal{N}$ and to put it in the input, i.e. the input would be a list of sources $i$ that are couples compound of a belief base and a weakening function: $\left\langle\varphi_{i}, \mathbf{\nabla}_{i}\right\rangle$ 
THEOREM 5. - Let $\Psi$ be a belief profile, and $\mu$ be a belief base. If $\Psi$ is non-empty and $\mu$ is consistent, then $\bigwedge \Psi_{\mathcal{N}}^{\mu} \wedge \mu$ is consistent and $\Psi_{\mathcal{N}}^{\mu}$ is reached for a finite number of rounds.

To prove that $\Psi_{\mathcal{N}}^{\mu}$ is reached for a finite number of rounds, it is sufficient to note that, at a given round, either $\Psi_{i}$ is consistent with $\mu$, so $\Psi_{\mathcal{N}}^{\mu}=\Psi_{i}$, and the process end, so we are done. Or $\Psi_{i}$ is not consistent with $\mu$, in this case there is an other round. From the defintion of the weakening of a profile, the resulting profile $\Psi_{i+1}$ is logically strictly weaker than $\Psi_{i}$, that means that each base of $\Psi_{i+1}$ is either logically equivalent to the corresponding base in $\Psi_{i}$, or logically strictly weaker than this base (see definition 2). Now just note that the logically weakest profile, is the one where each base is equivalent to $T$, and that this profile is achievable from every given belief profile by successive applications of the weakening function. Since we work in a finite propositional logic setting, this can be done by a finite number of rounds. Finally, note that this profile is consistent with every consistent integrity constraint $\mu$. So if the process described in definition 4 has not stopped before, it is guaranteed to give a result with this belief profile.

\section{Weakening and choice functions}

In order to define a particular Belief Game Model, we have to choose a choice function and a weakening function. We will give in this section some natural choices for these functions and see what are the resulting BGM operators.

\subsection{Weakening functions}

Let us first turn out to weakening functions. Can we find a "natural" one ? In fact it is a difficult task, since the exact choice of a weakening function depends on the expected behaviour for the Belief Game Model and depends also on the existence of some "preferential" information. But if we have no such additional information, we have at least two natural candidates : drastic weakening and dilation.

DEFINITION 6. - Let $\varphi$ be a belief base. The drastic weakening function forget all the information about one source, i.e. : $\nabla_{\top}(\varphi)=\top$.

This weakening function simply forget all the information in $\varphi$ !

After this rough function, let us see a more fine grained one. Let us first recall what is the Hamming's distance between interpretations (also called Dalal's distance [DAL 88]) since we will use it several times in this paper.

DEFINITION 7. - The Hamming distance between interpretations is the number of propositional symbols on which the two interpretations differ. Let $\omega$ and $\omega^{\prime}$ be two interpretations, then

$$
d_{H}\left(\omega, \omega^{\prime}\right)=\#\left(\left\{a \in \mathcal{P} \mid \omega(a) \neq \omega^{\prime}(a)\right\}\right)
$$


Then the dilation weakening function is defined as :

DEFINITION 8. - Let $\varphi$ be a belief base. The dilation weakening function is defined as :

$$
\bmod \left(\mathbf{\nabla}_{\delta}(\varphi)\right)=\left\{\omega \in \mathcal{W} \mid \exists \omega^{\prime} \models \varphi d_{H}\left(\omega, \omega^{\prime}\right) \leq 1\right\}
$$

This weakening function takes as models of the weakened base $\boldsymbol{\nabla}_{\delta}(\varphi)$, all the models that are at an Hamming distance less or equal to 1 from the models of $\varphi$, i.e. all the models that are in the base $\varphi$ and all the models that are achievable by flipping the truth value of one propositional symbol in a model of $\varphi$. This is close to the dilation operator used in morpho-logics [BLO 00, BLO 04].

\subsection{Choice functions}

Let us now turn to choice functions. The aim of this function is to determine the "losers", that are the sources that have to concede by weakening their beliefs at a given round.

One of the simplest choice functions is identity (denoted $g_{i d}$ ). It is not the expected behaviour for this function, but it can prove the rationality of our operators if, even in this case, we obtain a sensible merging.

We will focus on two families of choice functions. The first one is model-based, the second one is formula-based. We think that most of the sensible choice functions belong to one of those families.

\subsubsection{Model-based choice functions}

We will focus here on some modelizations of what can be called "social pressure", and can be viewed as a majority principle. Namely, at each round it is the "furthest" sources from the group that will concede. The exact choice of the meaning of "furthest" will fix the chosen operator for this family. Technically we will use a distance between belief bases and an aggregation function to evaluate the distance of a belief base with respect to the others.

We will start from the definition of the distance between two belief bases.

DEFINITION 9. - A (pseudo)distance ${ }^{3} d$ between two belief bases is a function $d$ : $\mathcal{L} \times \mathcal{L} \rightarrow \mathbb{I N}$ such that:

$-d\left(\varphi, \varphi^{\prime}\right)=0$ iff $\varphi \wedge \varphi^{\prime} \nvdash \perp$

$-d\left(\varphi, \varphi^{\prime}\right)=d\left(\varphi^{\prime}, \varphi\right)$

3. Remark that we miss an important property of distances: we have only $d\left(\varphi, \varphi^{\prime}\right)=0$ if $\varphi=$ $\varphi^{\prime}$, but not the only if part. Remark also that we do not require the triangular inequality. 
Two examples of such distances are :

$$
\begin{aligned}
& -d_{D}\left(\varphi, \varphi^{\prime}\right)= \begin{cases}0 & \text { if } \varphi \wedge \varphi^{\prime} \nvdash \perp \\
1 & \text { otherwise }\end{cases} \\
& -d_{H}\left(\varphi, \varphi^{\prime}\right)=\min _{\omega\left|=\varphi, \omega^{\prime}\right|=\varphi^{\prime}} d_{H}\left(\omega, \omega^{\prime}\right)
\end{aligned}
$$

DEFINITION 10. - An aggregation function is a total function $f$ associating a nonnegative integer to every finite tuple of nonnegative integers and verifying (nondecreasingness), (minimality) and (identity).

- if $x \leq y$, then $f\left(x_{1}, \ldots, x, \ldots, x_{n}\right) \leq f\left(x_{1}, \ldots, y, \ldots, x_{n}\right)$. (non-decreasingness)

$-f\left(x_{1}, \ldots, x_{n}\right)=0$ if and only if $x_{1}=\ldots=x_{n}=0$. (minimality)

- for every nonnegative integer $x, f(x)=x$. (identity)

We say that an aggregation function is symmetric if it also satisfies :

- For any permutation $\sigma, f\left(x_{1}, \ldots, x_{n}\right)=f\left(x_{\sigma(1)}, \ldots, x_{\sigma(n)}\right)$

(symmetry)

DEFINITION 11. - A model-based choice function $g^{d, h}$ is defined as :

$$
g^{d, h}(\Psi)=\left\{\varphi_{i} \in \Psi \mid h\left(d\left(\varphi_{i}, \varphi_{1}\right), \ldots, d\left(\varphi_{i}, \varphi_{n}\right)\right) \text { is maximal }\right\}
$$

where $h$ is an aggregation function, and $d$ is a distance between belief bases.

We say that the model-based choice function is symmetric if the aggregation function is symmetric.

We will focus on some specific aggregation functions in this paper, but we can use different aggregation functions here. In particular we will only focus on symmetrical aggregation functions in this paper (to fit with choice function requirements) but note that the definition allows non-symmetrical functions. This allows to define operators that are not anonymous, i.e. where each base has not the same importance. So one can use priorities (a weight or a pre-order on the sources) for denoting different level of reliability, different hierarchical importance, etc.

We will use in the following as examples of aggregation functions, two typical ones, the sum (noted $\Sigma$ ) and the maximum (noted max).

\subsubsection{Formula-based choice functions}

Not all interesting choice functions are captured in the definition given in the previous section. In particular, a lot of interesting choice functions can be defined by using maximal consistent subsets. Note, however that, conversely to usual formula-based merging operators [BAR 92, KON 00], we use multi-sets instead of simple sets.

DEFINITION 12. - Let MAXCONS $(\Psi)$ be the set of the maxcons of $\Psi$, i.e. the maximal (with respect to multi-set inclusion) consistent subsets of $\Psi$. Formally, $\operatorname{MAXCONS}(\Psi)$ is the set of all multi-sets $M$ such that:

$$
-M \sqsubseteq \Psi \text { and }
$$


- if $M \sqsubset M^{\prime} \sqsubseteq \Psi$, then $\bigwedge M^{\prime} \mid=\perp$.

DEFINITION 13. - A formula-based choice function $g^{m c}$ is a function of the set of the maxcons of $\Psi$ and the belief base, i.e. :

$$
g^{m c}(\Psi)=\left\{\varphi_{i} \in \Psi \mid h\left(\varphi_{i}, \operatorname{MAXCONS}(\Psi)\right) \text { is minimal }\right\}
$$

Examples of the use of maxcons are numerous, let us see two of them.

DEFINITION 14. -

$-h^{m c 1}(\varphi, \operatorname{MAXCONS}(\Psi))=\#(\{M \mid M \in \operatorname{MAXCONS}(\Psi)$ and $\varphi \in M\})$

$-h^{m c 2}(\varphi, \operatorname{MAXCONS}(\Psi))=\max (\{\#(M) \mid M \in \operatorname{MAXCONS}(\Psi)$ and $\varphi \in M\})$

The first function computes the number of maxcons the belief base belongs to. The second function computes the size of the biggest maxcons the belief base belongs to.

We will note $g^{m c 1}$ (respectively $g^{m c 2}$ ) the formula-based choice function that use $h^{m c 1}$ (resp. $\left.h^{m c 2}\right)$.

\section{Instantiating the BGM framework}

In this section we will try to illustrate how interesting the defined Belief Game Model framework is by giving several examples. We will first see some of the simplest operators that we can define with this framework. Then we will illustrate the behaviour of more complex operators on a typical merging example.

\subsection{Some simple examples}

Let us first see what operators are obtained with the simplest weakening and choice functions (that means that we will either choose the weakening function to be the drastic one, or the choice function to be identity).

$-\left\langle g_{i d}, \mathbf{\nabla}_{\top}\right\rangle:$ In this case the belief base result of the BGM on $\Psi$ under the constraint $\mu$ is the conjunction of all the bases of the profile with the integrity constraints $(\bigwedge \Psi \wedge \mu)$ if this conjunction is consistent, and $\mu$ otherwise. This operator is called the basic merging operator [KON 99].

$-\left\langle g_{i d}, \mathbf{\nabla}_{\delta}\right\rangle$ : In this case, at each step of the game, each source weakens using dilation. This gives the well known model-based merging operator $\triangle^{d_{H} \text {, max }}$ defined in [REV 93, REV 97, KON 02a].

$-\left\langle g^{d_{D}, \Sigma}, \nabla_{\top}\right\rangle:$ Here, the result is the cardinality-maximal consistent subset of $\Psi$ if it is unique and consistent with the constraints $\mu$, and it is simply $\mu$ otherwise. This operator is a new one. It is interesting since it can be viewed as a generalized conjunction : it gives the conjunction of all the bases and the constraints if it is consistent, 
but if it is not, it tries to find the result by doing the least number of repairs (forget one belief base) of the belief profile. If there is no ambiguity on the correction (i.e. a unique cardinality-maxcons), then it accepts it as the result.

$-\left\langle g^{d_{D}, \max }, \mathbf{\nabla}_{\top}\right\rangle$ : This operator gives as result the conjunction of all the formulas that belong to all maxcons (also called free formulas in [BEN 97, BEN 99]) and the integrity constraints if it is consistent, and $\mu$ otherwise.

$-\left\langle g^{m c 1}, \nabla_{\top}\right\rangle$ : This operator gives the conjunction of the formulas that belong to the maximum number of maxcons and the integrity constraints if consistent, and $\mu$ otherwise.

$-\left\langle g^{m c 2}, \mathbf{\nabla}_{\top}\right\rangle:$ In this case, the belief base result of the merging is the conjunction of the belief bases that belong to the biggest maxcons for cardinality and the integrity constraints if consistent, and $\mu$ otherwise.

These operators are not logically independent, some of them are logically stronger than others, as stated in the following proposition.

THEOREM 15. - In figure 1 an arrow between an operator $A$ and an operator $B(A \longrightarrow B)$ means that operator $A$ is logically stronger ${ }^{4}$ (or less cautious) than operator B. Results obtained by transitivity are not represented.

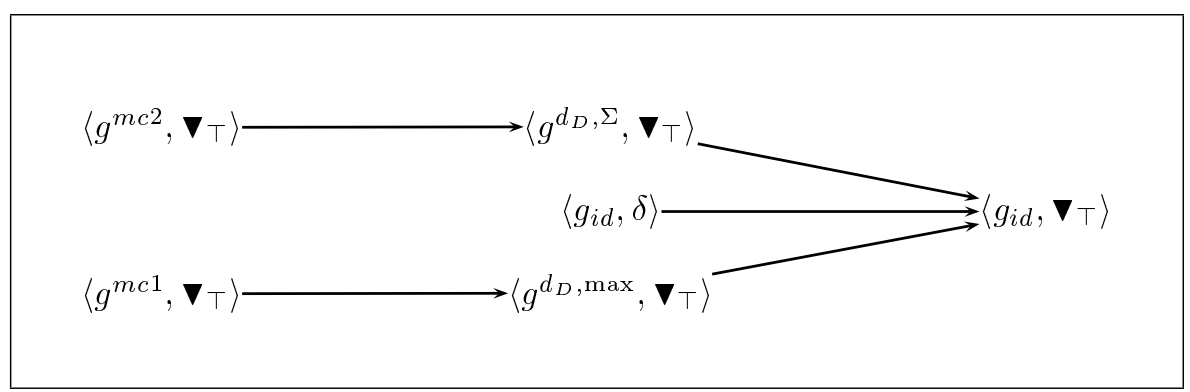

Figure 1. Cautiousness

\subsection{An example}

We will see on an example [REV 97], what is the behaviour or some BGM operators, namely the operators $\left\langle g^{d_{H}, h^{\Sigma}}, \mathbf{\nabla}_{\delta}\right\rangle,\left\langle g^{d_{H}, h^{\max }}, \mathbf{\nabla}_{\delta}\right\rangle,\left\langle g^{m c 1}, \mathbf{\nabla}_{\delta}\right\rangle$ and $\left\langle g^{m c 2}, \mathbf{\nabla}_{\delta}\right\rangle$. Here is the example: There are three sources $\Psi=\left\{\varphi_{1}, \varphi_{2}, \varphi_{3}\right\}$ with the following belief bases $\operatorname{Mod}\left(\varphi_{1}\right)=\{(1,0,0),(0,0,1),(1,0,1)\}, \operatorname{Mod}\left(\varphi_{2}\right)=$ $\{(0,1,0),(0,0,1)\}, \operatorname{Mod}\left(\varphi_{3}\right)=\{(1,1,1)\}$. There are no constraints on the result, so $\mu=\top$.

4. An operator $A$ is logically stronger than an operator $B$ iff for all profile $\Psi, A(\Psi) \vdash B(\Psi)$, where $A(\Psi)$ denotes the belief base result of the BGM $A$ on the profile $\Psi$. 
$-\left\langle g^{d_{H}, h^{\Sigma}}, \mathbf{\nabla}_{\delta}\right\rangle:$ As $\Psi$ is not consistent, let us do the first round. $d\left(\varphi_{1}, \varphi_{2}\right)=$ $0, d\left(\varphi_{1}, \varphi_{3}\right)=1, d\left(\varphi_{2}, \varphi_{3}\right)=2$. So $h_{\Psi}^{\Sigma}\left(\varphi_{1}\right)=1, h_{\Psi}^{\Sigma}\left(\varphi_{2}\right)=2$, $h_{\Psi}^{\Sigma}\left(\varphi_{3}\right)=3$. That gives $g^{d_{H}, h^{\Sigma}}(\Psi)=\left\{\varphi_{3}\right\}$. So $\varphi_{3}$ is $\operatorname{replaced}^{5}$ by $\mathbf{\nabla}_{\delta}\left(\varphi_{3}\right)=$ form $(\{(1,1,1),(1,1,0),(1,0,1),(0,1,1)\})$. We have not yet reached a consistent $\Psi$, so let us do a further round. Let us first compute the new distances. $d\left(\varphi_{1}, \varphi_{2}\right)=0, d\left(\varphi_{1}, \varphi_{3}\right)=0, d\left(\varphi_{2}, \varphi_{3}\right)=1$. So $h_{\Psi}^{\Sigma}\left(\varphi_{1}\right)=0$, $h_{\Psi}^{\Sigma}\left(\varphi_{2}\right)=1, h_{\Psi}^{\Sigma}\left(\varphi_{3}\right)=1$. That gives $g^{d_{H}, h^{\Sigma}}(\Psi)=\left\{\varphi_{2}, \varphi_{3}\right\}$. So $\varphi_{2}$ is replaced by $\mathbf{\nabla}_{\delta}\left(\varphi_{2}\right)=$ form $(\{(0,1,0),(0,0,1),(1,1,0),(0,0,0),(0,1,1),(1,0,1)\})$, and $\varphi_{3}$ is replaced by $\mathbf{\nabla}_{\delta}\left(\varphi_{3}\right)=$ form $(\{(1,1,1),(1,1,0),(1,0,1),(0,1,1),(0$, $1,0),(1,0,0),(0,0,1)\})$. We have reached a consistent belief profile, so the result is $\left.\operatorname{Mod}\left(\Psi_{\left\langle g^{d}, h^{\Sigma}\right.}, \mathbf{v}_{\delta}\right\rangle\right)=\{(0,0,1),(1,0,1)\}$.

$-\left\langle g^{d_{H}, h^{\max }}, \mathbf{\nabla}_{\delta}\right\rangle:$ As $\Psi$ is not consistent, let us do the first round. $d\left(\varphi_{1}, \varphi_{2}\right)=0$, $d\left(\varphi_{1}, \varphi_{3}\right)=1, d\left(\varphi_{2}, \varphi_{3}\right)=2$. So $h_{\Psi}^{\max }\left(\varphi_{1}\right)=1, h_{\Psi}^{\max }\left(\varphi_{2}\right)=2, h_{\Psi}^{\max }\left(\varphi_{3}\right)=$ 2. That gives $g^{d_{H}, h^{\text {max }}}(\Psi)=\left\{\varphi_{2}, \varphi_{3}\right\}$. So $\varphi_{2}$ is replaced by $\mathbf{\nabla}_{\delta}\left(\varphi_{2}\right)=$ form $(\{(0,1,0),(0,0,1),(1,1,0),(0,0,0),(0,1,1),(1,0,1)\})$, and $\varphi_{3}$ is replaced by $\mathbf{\nabla}_{\delta}\left(\varphi_{3}\right)=\operatorname{form}(\{(1,1,1),(1,1,0),(1,0,1),(0,1,1)\})$. The obtained profile is consistent, so the result is $\operatorname{Mod}\left(\Psi_{\left\langle g^{d}, h^{\max }, \mathbf{v}_{\delta}\right\rangle}\right)=\{(1,0,1)\}$.

$-\left\langle g^{m c 1}, \mathbf{v}_{\delta}\right\rangle: \Psi$ is not consistent, and $\operatorname{maXCONS}(\Psi)=\left\{\left\{\varphi_{1}, \varphi_{2}\right\},\left\{\varphi_{3}\right\}\right\}$. So $h_{\Psi}^{m c 1}\left(\varphi_{1}\right)=h_{\Psi}^{m c 1}\left(\varphi_{2}\right)=h_{\Psi}^{m c 1}\left(\varphi_{3}\right)=1$, and $g^{m c 1}(\Psi)=$ $\Psi$. So we weaken the three bases, which gives respectively $\nabla_{\delta}\left(\varphi_{1}\right)=$ form $(\{(1,0,0),(0,0,1),(1,0,1),(0,0,0),(1,1,0),(0,1,1),(1,1,1)\}), \mathbf{\nabla}_{\delta}\left(\varphi_{2}\right)=$ form $(\{(0,1,0),(0,0,1),(1,1,0),(0,0,0),(0,1,1),(1,0,1)\})$, and $\mathbf{\nabla}_{\delta}\left(\varphi_{3}\right)=$ form $(\{(1,1,1),(1,1,0),(1,0,1),(0,1,1)\})$. This belief profile is consistent, and the resulting base is $\operatorname{Mod}\left(\Psi_{\left\langle g^{m c 1}, \mathbf{v}_{\delta}\right\rangle}\right)=\{(1,0,1),(1,1,0),(0,1,1)\}$.

$-\left\langle g^{m c 2}, \mathbf{\nabla}_{\delta}\right\rangle: \Psi$ is not consistent, and we have $\operatorname{MaXCONS}(\Psi)=$ $\left\{\left\{\varphi_{1}, \varphi_{2}\right\},\left\{\varphi_{3}\right\}\right\}$. So $h_{\Psi}^{m c 2}\left(\varphi_{1}\right)=h_{\Psi}^{m c 2}\left(\varphi_{2}\right)=2$ and $h_{\Psi}^{m c 2}\left(\varphi_{3}\right)=$ 1 , and $g^{m c 2}(\Psi)=\left\{\varphi_{3}\right\}$. So $\varphi_{3}$ is replaced by $\nabla_{\delta}\left(\varphi_{3}\right)=$ form $(\{(1,1,1),(1,1,0),(1,0,1),(0,1,1)\})$. The belief profile is still not consistent, so we need one more round. Now we have $\operatorname{MAXCONS}(\Psi)=$ $\left\{\left\{\varphi_{1}, \varphi_{2}\right\},\left\{\varphi_{1}, \varphi_{3}\right\}\right\}$. So $h_{\Psi}^{m c 2}\left(\varphi_{1}\right)=h_{\Psi}^{m c 2}\left(\varphi_{2}\right)=h_{\Psi}^{m c 2}\left(\varphi_{3}\right)=2$, and $g^{m c 2}(\Psi)=\Psi$. So we weaken the three bases, which gives respectively $\nabla_{\delta}\left(\varphi_{1}\right)=\operatorname{form}(\{(1,0,0),(0,0,1),(1,0,1),(0,0,0),(1,1,0),(0$, $1,1),(1,1,1)\}), \mathbf{\nabla}_{\delta}\left(\varphi_{2}\right)=\operatorname{form}(\{(0,1,0),(0,0,1),(1,1,0),(0,0,0),(0,1,1)$, $(1,0,1)\})$, and $\mathbf{\nabla}_{\delta}\left(\varphi_{3}\right)=\operatorname{form}(\{(1,1,1),(1,1,0),(1,0,1),(0,1,1)\})$. The belief profile is consistent, and the resulting base is $\operatorname{Mod}\left(\Psi_{\left\langle g^{m c 2},\right.}, \mathbf{v}_{\delta}\right\rangle=$ $\{(0,0,1),(1,0,1),(1,1,0),(0,1,1)\}$.

As we can note, on this example the four operators give different (non trivial) results. As all these operators take dilation as weakening functions, we sometimes have the interpretation $(1,1,0)$ as model of the base result of the merging, whereas

5. In order to avoid unnecessary notations, we do not use subscripts to denote the different weakening steps of the bases, we simply replace the belief bases by their weakened counterparts. Hopefully, it can not lead to confusions. 
it is a model of none of the initial belief bases. This means that, conversely to usual formula-based merging operators [BAR 92, KON 00, KON 04], the result of the BGM does not (always) imply the disjunction of the belief bases of the profile.

\section{Logical properties}

Some work in belief merging aims at finding sets of axiomatic properties operators may exhibit in order to ensure the expected behaviour [REV 93, REV 97, LIB 98, KON 98, KON 99, KON 02b]. We focus here on the characterization of Integrity Constraints (IC) merging operators [KON 99, KON 02a].

DEFINITION 16 (IC MERGING OPERATORS). - $-\triangle$ is an IC merging operator if and only if it satisfies the following properties:

(IC0) $\triangle_{\mu}(\Psi) \models \mu$

(IC1) If $\mu$ is consistent, then $\triangle_{\mu}(\Psi)$ is consistent

(IC2) If $\bigwedge \Psi$ is consistent with $\mu$, then $\triangle_{\mu}(\Psi) \equiv \wedge \Psi \wedge \mu$

(IC3) If $\Psi_{1} \equiv \Psi_{2}$ and $\mu_{1} \equiv \mu_{2}$, then $\triangle_{\mu_{1}}\left(\Psi_{1}\right) \equiv \triangle_{\mu_{2}}\left(\Psi_{2}\right)$

(IC4) If $\varphi_{1}=\mu$ and $\varphi_{2} \models \mu$, then $\triangle_{\mu}\left(\left\{\varphi_{1}, \varphi_{2}\right\}\right) \wedge \varphi_{1}$ is consistent if and only if $\triangle_{\mu}\left(\left\{\varphi_{1}, \varphi_{2}\right\}\right) \wedge \varphi_{2}$ is consistent

(IC5) $\triangle_{\mu}\left(\Psi_{1}\right) \wedge \triangle_{\mu}\left(\Psi_{2}\right) \models \triangle_{\mu}\left(\Psi_{1} \sqcup \Psi_{2}\right)$

(IC6) If $\triangle_{\mu}\left(\Psi_{1}\right) \wedge \triangle_{\mu}\left(\Psi_{2}\right)$ is consistent, then $\triangle_{\mu}\left(\Psi_{1} \sqcup \Psi_{2}\right) \models \triangle_{\mu}\left(\Psi_{1}\right) \wedge \triangle_{\mu}\left(\Psi_{2}\right)$

(IC7) $\triangle_{\mu_{1}}(\Psi) \wedge \mu_{2} \models \triangle_{\mu_{1} \wedge \mu_{2}}(\Psi)$

(IC8) If $\triangle_{\mu_{1}}(\Psi) \wedge \mu_{2}$ is consistent, then $\triangle_{\mu_{1} \wedge \mu_{2}}(\Psi) \models \triangle_{\mu_{1}}(\Psi)$

The intuitive meaning of the properties is the following: (ICO) ensures that the result of merging satisfies the integrity constraints. (IC1) states that, if the integrity constraints are consistent, then the result of merging will be consistent. (IC2) states that if possible, the result of merging is simply the conjunction of the belief bases with the integrity constraints. (IC3) is the principle of irrelevance of syntax : the result of merging has to depend only on the expressed opinions and not on their syntactical presentation. (IC4) is a fairness postulate meaning that the result of merging of two belief bases should not give preference to one of them (if it is consistent with one of both, it has to be consistent with the other one.) It is a symmetry condition, that aims to rule out operators that can give priority to one of the bases. (IC5) expresses the following idea: if belief profiles are viewed as expressing the beliefs of the members of a group, then if $\Psi_{1}$ (corresponding to a first group) compromises on a set of alternatives which $A$ belongs to, and $\Psi_{2}$ (corresponding to a second group) compromises on another set of alternatives which contains $A$ too, then $A$ has to be in the chosen 
alternatives if we join the two groups. (IC5) and (IC6) together state that if one could find two subgroups which agree on at least one alternative, then the result of the global merging will be exactly those alternatives the two groups agree on. (IC7) and (IC8) state that the notion of closeness is well-behaved, i.e. that an alternative that is preferred among the possible alternatives $\left(\mu_{1}\right)$, will remain preferred if one restricts the possible choices $\left(\mu_{1} \wedge \mu_{2}\right)$. For more explanations on those properties see [KON 02a].

So, let us see now what are the properties of BGM operators.

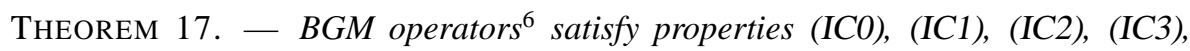
(IC7), (IC8). They do not necessarily satisfy properties (IC4), (IC5), (IC6).

PROOF. -

(IC0) is satisfied by definition (see definition 4), since the result of the BGM process is the base $\wedge \Psi_{\mathcal{N}}^{\mu} \wedge \mu$, so it implies $\mu$.

(IC1) is satisfied by definition (see theorem 5).

(IC2) by definition of the BGM (see definition 4), if $\bigwedge \Psi$ is consistent with $\mu$, then the halting condition is satisfied before any weakening round, so $\Psi_{\mathcal{N}}^{\mu}=\Psi$, and $\bigwedge \Psi_{\mathcal{N}}^{\mu} \wedge \mu \equiv \bigwedge \Psi \wedge \mu$

(IC3) is a straighforward consequence of the third condition of the weakening functions, and of the last condition of the choice functions.

(IC7) and (IC8) are satisfied. First note that if $\triangle_{\mu_{1}}(\Psi) \wedge \mu_{2}$ is not consistent, then $\triangle_{\mu_{1}}(\Psi) \wedge \mu_{2} \models \triangle_{\mu_{1} \wedge \mu_{2}}(\Psi)$, so (IC7) is trivially satisfied. Now, in the case $\triangle_{\mu_{1}}(\Psi) \wedge$ $\mu_{2}$ consistent, let us prove (IC7) and (IC8), that is $\triangle_{\mu_{1} \wedge \mu_{2}}(\Psi) \equiv \triangle_{\mu_{1}}(\Psi) \wedge \mu_{2}$. As $\triangle_{\mu_{1}}(\Psi)=\Psi_{\mathcal{N}}^{\mu_{1}} \wedge \mu_{1}$, and by definition $4, \Psi_{\mathcal{N}}^{\mu_{1}}$ is the first profile $\Psi_{i}$ such that $\bigwedge \Psi_{i} \wedge \mu_{1}$ is consistent. As we also know that, by hypothesis, $\bigwedge \Psi_{i} \wedge \mu_{1} \wedge \mu_{2}$ is consistent, $\Psi_{i}$ is also the first profile such that $\bigwedge \Psi_{i} \wedge \mu_{1} \wedge \mu_{2}$ is consistent. By definition it means that $\Psi_{\mathcal{N}}^{\mu_{1} \wedge \mu_{2}}=\Psi_{i}$. So we get that $\Psi_{\mathcal{N}}^{\mu_{1}}=\Psi_{\mathcal{N}}^{\mu_{1} \wedge \mu_{2}}$. From that we get $\left(\Psi_{\mathcal{N}}^{\mu_{1}} \wedge \mu_{1}\right) \wedge \mu_{2}=\Psi_{\mathcal{N}}^{\mu_{1}} \wedge_{\mu_{2}} \wedge \mu_{1} \wedge \mu_{2}$, which is exactly $\triangle_{\mu_{1}}(\Psi) \wedge \mu_{2}=\triangle_{\mu_{1} \wedge \mu_{2}}(\Psi)$.

So, as stated in the previous proposition, BGM operators do not fit all properties of IC merging operators. On the other hand, we know for example that the operator $\left\langle g_{i d}, \mathbf{\nabla}_{\delta}\right\rangle=\triangle^{d_{H}, \max }$ satisfies also (IC4), (IC5) [KON 02a]. So the question is to know if we can ensure more logical properties by making some restrictions on the weakening and/or the choice functions.

A first remark is that (IC4) cannot be proved to hold for any BGM operator, but it is satisfied for all the particular operators we have defined in this paper.

THEOREM 18. - If the weakening function is dilation or drastic weakening, and if the choice function is a symmetric model-based choice function or the formula-based choice function $g^{m c 1}$ or $g^{m c 2}$, then the BGM operator satisfies (IC4).

6. Defined from any choice function and any weakening function. 
PROOF. - First note that for all those operators if the two bases are consistent with the constraints (i.e. $\varphi_{1} \wedge \varphi_{2} \wedge \mu$ ), then the result of the merging is (from (IC2)) $\triangle_{\mu}\left(\left\{\varphi_{1}, \varphi_{2}\right\}\right)=\varphi_{1} \wedge \varphi_{2} \wedge \mu$, so (IC4) is trivially satisfied. The interesting part of (IC4) is when $\varphi_{1} \wedge \varphi_{2} \wedge \mu$ is not consistent.

If the choice function is a symmetric model-based choice function, then simply note that the distance used to define the model-based functions are symmetric by definition, so $d\left(\varphi, \varphi^{\prime}\right)=d\left(\varphi^{\prime}, \varphi\right)$. Let note also that by definition of the distance $d(\varphi, \varphi)=d\left(\varphi^{\prime}, \varphi^{\prime}\right)=0$. So, now, using the symmetry condition of the aggregation function, it is easy to show that $h\left(d(\varphi, \varphi), d\left(\varphi, \varphi^{\prime}\right)\right)=h\left(d\left(\varphi^{\prime}, \varphi\right), d\left(\varphi^{\prime}, \varphi^{\prime}\right)\right)$, that means that $\varphi$ and $\varphi^{\prime}$ are always both maximal, and that they always both have to be weakened. If we use drastic weakening as the weakening function, we have that $\varphi$ and $\varphi^{\prime}$ are replaced by $\top$ and $\top$. So the result of the merging is consistent with $\varphi$ and $\varphi^{\prime}$. So (IC4) is satisfied. If the weakening function is dilation, then when we weaken $\varphi$ (resp. $\varphi^{\prime}$ ) for the $n^{t h}$ time, we get as the result the base $\varphi^{n}$ (resp. $\varphi^{\prime n}$ ) that is the formula (up to logical equivalence) whose models are the ones that are at a (Dalal) distance less or equal to $n$ to models of $\varphi$ (resp. $\varphi^{\prime}$ ). So, let note the final profile of the BGM $\Psi_{i}=\left\{\varphi^{i}, \varphi^{\prime i}\right\}$, if $\bigwedge \Psi_{i} \wedge \varphi$ is consistent, it means that $d_{H}\left(\varphi^{\prime i}, \varphi\right)=0$, but by symmetry it implies that $d_{H}\left(\varphi^{i}, \varphi^{\prime}\right)=0$, so (recall also that, by definition of dilation $d_{H}\left(\varphi^{i}, \varphi\right)=0$ and $\left.d_{H}\left(\varphi^{\prime i}, \varphi^{\prime}\right)=0\right)$ that $\wedge \Psi_{i} \wedge \varphi^{\prime}$ is consistent. So (IC4) is satisfied.

If the choice function is $g^{m c 1}$ or $g^{m c 2}$, let us note that the maximal consistent sets are $\{\varphi\}$ and $\left\{\varphi^{\prime}\right\}$, so the $\varphi$ and $\varphi^{\prime}$ are both minimal, and that they always both have to be weakened. If we use drastic weakening as the weakening function, we have that $\varphi$ and $\varphi^{\prime}$ are replaced by $\top$ and $T$. So the result of the merging is consistent with $\varphi$ and $\varphi^{\prime}$. So (IC4) is satisfied. If the weakening function is dilation, we use the same symmetry argument as for symmetric model-based choice functions.

The property (IC5) can also be recovered for some BGM operators, but (IC6) seems hardly recoverable. Those two properties ((IC5) and (IC6)) are important for classical merging operators, so we can wonder if the BGM operators missing those properties can still be called "merging" operators. One answer to this is that the BGM operators aim at focusing on the interaction between the beliefs of the sources, so it seems natural to loose property (IC6). Indeed, whereas classical merging operators aim at giving the result of the merging process in an ideal framework, BGM operators seem more adequately reflect the behaviour of a real multi-source merging process.

Another important logical link to be underlined is the relationship between BGM operators and AGM belief revision operators [ALC 85, GÄR 88, KAT 91, GÄR 92]. Belief revision aims to make the minimum change in a belief base in order to take into account new information that is more reliable than the current belief base (and that usually contradicts the current belief base). Technically those operators can be described as follows : until the belief base is consistent with the new item of information 
(seen as an integrity constraint) then weaken the belief base ${ }^{7}$. Stated this way, we can immediately see the parallel with BGM operators since they are described as follows : until the belief profile is consistent with the constraint then weaken some belief bases. The following result shows more formally that, as explained above, BGM operators can be seen as a direct generalization of AGM belief revision operators.

THEOREM 19. - Let $\mathcal{N}=\langle g, \mathbf{\nabla}\rangle$ be a BGM operator. Let $\varphi$ and $\mu$ be two belief bases. The operator $\circ$ defined as $\varphi \circ \mu=\mathcal{N}_{\mu}(\{\varphi\})$ is an AGM belief revision operator (i.e. it satisfies properties (R1-R6) of [KAT 91]).

PROOF. - It is easy to see that if we restrict belief profiles $\Psi$ to singletons $\{\varphi\}$, then postulates (IC0), (IC1), (IC2), (IC3), (IC7), (IC8) directly translate to (R1-R6). After that the proof follows from theorem 17.

In particular, we have that each BGM using the dilation weakening function is a generalization of Dalal's revision operator [DAL 88].

Finally let us see another cardinality restriction on the belief profile.

THEOREM 20. - Let $\mathcal{N}=\left\langle g^{d, h}, \mathbf{\nabla}_{\delta}\right\rangle$ be a BGM operator defined from a symmetric model-based choice function and dilation weakening function. Let $\varphi_{1}, \varphi_{2}$ and $\mu$ be three belief bases, then the operator $\mathcal{N}_{\mu}\left(\left\{\varphi_{1}, \varphi_{2}\right\}\right)$ is the model-based merging operator $\triangle_{\mu}^{d_{H}, \max }\left(\left\{\varphi_{1}, \varphi_{2}\right\}\right)$ [KON 02a].

Note that the previous result holds only when we merge two belief bases.

\section{Comparison between BGM and BNM}

In this section we will mainly compare our proposal with Booth's Belief Negotiation Model (BNM) [BOO 02]. Let us first briefly recall Booth's proposal.

Belief profiles in this framework are no longer multi-sets but vectors of belief bases, noted $\vec{\Psi}$. Let us note $\overrightarrow{\mathcal{E}}$ the set of belief profiles, and let us note $\vec{\Sigma}$ the set of all sequences (vectors) of belief profiles, and $\vec{\sigma}$ one element of this set. When $\vec{X}$ is a vector, we will note $\vec{X}^{i}$ the $i$ th element of the vector and $\vec{X}^{m}$ the last element of the vector.

So a sequence of belief profiles $\vec{\sigma}$ is of the form $\vec{\sigma}=\left\{\vec{\Psi}_{1}, \ldots, \vec{\Psi}_{n}\right\}$, with each $\vec{\Psi}_{i}$ being a vector of belief bases, i.e. $\vec{\Psi}_{i}=\left(\varphi_{i, 1}, \ldots, \varphi_{i, n_{i}}\right)$. And, for example, the notation $\vec{\sigma}^{3}$ stands for $\vec{\Psi}_{3}$, and the notation $\vec{\sigma}^{m}$ stands for $\vec{\Psi}_{n}$.

Then a BNM negotiation (choice) function is defined as :

DEFINITION 21. - A BNM negotiation function is a function $g^{B N M}: \vec{\Sigma} \rightarrow \overrightarrow{\mathcal{E}}$ such that:

7. It is the intuitive meaning behind Katsuno and Mendelzon representation theorem in terms of faithful assignments [KAT 91]. 


$$
\begin{aligned}
& -g^{B N M}(\vec{\sigma}) \sqsubseteq \vec{\sigma}^{m} \\
& -g^{B N M}(\vec{\sigma}) \neq \emptyset \\
& - \text { If } \varphi_{i} \in g^{B N M}(\vec{\sigma}), \text { then } \varphi_{i} \not \equiv \top
\end{aligned}
$$

And a BNM weakening function is defined as :

DEFINITION 22. - A BNM weakening function is a function $\boldsymbol{\nabla}^{B N M}: \vec{\Sigma} \rightarrow \overrightarrow{\mathcal{E}}$ such that:

$-\left(\vec{\sigma}^{m}\right)^{i} \vdash \boldsymbol{\nabla}^{B N M}(\vec{\sigma})^{i}$

- If $\left(\vec{\sigma}^{m}\right)^{i} \equiv \mathbf{\nabla}^{B N M}(\vec{\sigma})^{i}$, then $\left(\vec{\sigma}^{m}\right)^{i} \equiv \top$

Finally the solution to a BNM is defined as :

DEFINITION 23. - The solution to a belief profile $\vec{\Psi}$ for a Belief Negotiation Model $\mathcal{N}^{B N M}=\left\langle g^{B N M}, \nabla^{B N M}\right\rangle$ under the integrity constraints $\mu$, noted $\mathcal{N}_{\mu}^{B N M}(\Psi)$, is given by the function $f^{\mathcal{N}}: \overrightarrow{\mathcal{E}} \rightarrow \vec{\Sigma}$ defined as:

$$
-f^{\mathcal{N}}(\vec{\Psi})=\vec{\sigma}=\left(\vec{\Psi}_{0}, \ldots, \vec{\Psi}_{k}\right)
$$

with $\vec{\Psi}_{0}=\vec{\Psi}, k$ is the smallest integer such that $\bigwedge \vec{\Psi}_{k} \wedge \mu$ is consistent, and for each $0 \leq j<k$ we have $\left(\vec{\sigma}_{j}\right.$ denotes $\left.\left(\vec{\Psi}_{0}, \ldots, \vec{\Psi}_{j}\right)\right)$ :

$$
\left(\vec{\Psi}_{j+1}\right)^{i}= \begin{cases}\nabla^{B N M}\left(\vec{\sigma}_{j}\right)^{i} & \text { if }\left(\vec{\Psi}_{j}\right)^{i} \in g^{B N M}\left(\vec{\sigma}_{j}\right) \\ \left(\vec{\Psi}_{j}\right)^{i} & \text { otherwise }\end{cases}
$$

Finally, the belief base result of the BNM is $\wedge \vec{\Psi}_{k} \wedge \mu$.

We change some notation, in order to show the closeness with our present work. For the original presentation and detailed explanations on the definitions see [BOO 02 , BOO 01].

The main differences between BNM and BGM are :

- BNM's definition of belief profile as vectors allows us to speak about sources separately. So when there are two identical belief bases in the belief profile, it is possible to weaken only one of these bases. This is not possible in the BGM framework.

- The BNM negotiation function takes as input the whole negotiation history from the initial belief profile. So it is possible to implement a negotiation process such that each source weakens after the previous one (for example, source 1 , then source $2, \ldots$ ), or such that we prevent a source to weaken two times successively. The BGM choice functions are more Markovian, taking only into account the current belief profile.

- Similarly, the BNM weakening function also takes as input the whole negotiation history. It allows us to weaken differently two identical belief bases obtained at different rounds or to weaken differently two identical belief bases of the same belief profile.

- According to the previous items ideas, the irrelevance of syntax condition of the BGM weakening function, and the anonymity condition of the BGM choice function 
are not required in the BNM framework.

The main difference between Booth's proposal and our is that Booth's takes the sources as candidates to weakening, whereas we restrict ourselves to "points of view" (logical content of the bases). That means that in Booth's if one source has to weaken, it can be the case that another source with exactly the same beliefs do not have to weaken too. Our proposal adds more anonymity by saying that only beliefs decide who has to weaken, not the identity of one source. Similarly, the choice functions are more "Markovian" in our framework than in Booth's one. We think that those hypothesis are more realistic (and necessary) on a belief merging point of view. Whereas Booth's framework allows us to model more generalized negotiation frameworks, where one can decide for example that each source has to weaken one after the other. So, despite the closeness of the models, and the objective fact that our proposal is a particular case of Booth's one (i.e. each of our operators can be defined in Booth's framework), the intended applications of those two frameworks are quite different. And the particular properties achieved by adding those restrictions shows that this framework forms a consistent family of merging operators. It explains why it is worth focusing on the model we have defined.

A last difference is that, in this paper, we are interested on the result of the process (as a belief base), whereas BNM framework aims at studying the resulting profile, in connection with a notion of "social contraction". See [BOO 02] for a study of logical properties for social contraction.

An additional contribution of this work is to give examples of purely propositional logic BNM operators. In [BOO 02], Booth propose two examples of BNM, both working on ordinal conditional functions (OCF) [SPO 88], but none on a propositional belief base. So this work can be seen as an investigation of what kind of operators this definition can give on propositional belief bases (through adding additional requirements).

\section{Conclusion}

We have proposed in this paper a new family of belief merging operators, that we call Belief Game Model (BGM) operators. The hypothesis for those operators is that all the sources are a priori reliable, or that we know that some sources are less reliable than the others, but without knowing which ones. This hypothesis leads to choosing a majority approach, justified by Condorcet's Jury Theorem. The idea behind the Belief Game Model is simple : Until a coherent set of sources is reached, at each round a contest is organized to find out the weakest sources, then those sources have to concede (weaken their point of view). This idea leads to numerous new interesting operators and opens new perspectives for belief merging. Some existing operators are also recovered as particular cases.

Not surprisingly, the operators defined do not satisfy all logical properties proposed for IC merging operators. The reason is that those logical properties aim at 
giving constraints on the result of the merging in an ideal framework, whereas BGM operators aim at describing more accurately what can happen in a real multi-source environment. So usual IC merging operators can be seen as a normative approach to merging. They show the way to a purely logical result. Conversely, BGM operators adopt a descriptive approach to merging, taking into account the interaction between the sources. They try to simulate more adequately what can happen in a group-decision process. So they are maybe more realistic.

This paper mainly aims to introduce BGM operators, but it provides several open questions that are left for further research.

The first one is about the definition of BGM operators and the computation of the result. We give an iterative definition of BGM operators, that leads to an iterative computation of the result. The question is to know if we can find a non-iterative equivalent definition. We know that some simple operators can be defined non-iteratively. But the question is to know if all operators or a non-trivial subclass of them are also definable non-iteratively.

Another open question is about the logical characterization of this family. In this paper we study the logical properties of this family with respect to the general definition of IC merging operators. The question is to know if we can find a set of logical properties that characterizes BGM operators.

Finally, we have recently studied the strategy-proofness of the usual propositional merging operators, showing that most of them are not strategy-proof [EVE 04]. And we have exhibited several restrictions on which strategy-proofness can be achieved. So an interesting question is to compare the strategy-proofness of BGM operators with the one of the classical merging operators.

\section{Acknowledgements}

The author wish to thank Richard Booth for hepful discussions and for its comments on a previous version of this paper, the anonymous referees for their interesting comments and Anthony Hunter for its help. The author has been supported by the IUT de Lens, the Université d'Artois, the Région Nord/Pas-de-Calais under the TACT-TIC project, and by the European Community FEDER Program.

\section{References}

[AlC 85] Alchourrón C. E., Gärdenfors P., Makinson D., "On the logic of theory change: Partial meet contraction and revision functions", Journal of Symbolic Logic, vol. 50, 1985, p. 510-530.

[BAR 92] Baral C., Kraus S., Minker J., Subrahmanian V. S., "Combining knowledge bases consisting of first-order theories", Computational Intelligence, vol. 8, num. 1, 1992, p. 45-71. 
[BEN 97] Benferhat S., Dubois D., Prade H., "Some syntactic approaches to the handling of inconsistent knowledge bases: a comparative study, part 1: the flat case", Studia Logica, vol. 58, 1997, p. 17-45.

[Ben 98a] Benferhat S., Dubois D., Lang J., Prade H., SAfFioti A., Smets P., “A general approach for inconsistency handling and merging information in prioritized knowledge bases", Proceedings of the Sixth International Conference on Principles of Knowledge Representation and Reasoning (KR'98), 1998, p. 466-477.

[BEN 98b] Benferhat S., Dubois D., Prade H., "Some syntactic approaches to the handling of inconsistent knowledge bases: A comparative study. Part II: The prioritized case", ORLOWSKA E., Ed., Logic at work, vol. 24 of Physica-Verlag, Heidelberg, 1998, p. 473511.

[BEN 99] Benferhat S., Dubois D., Prade H., "An overview of inconsistency-tolerant inferences in prioritized knowledge bases", Fuzzy Sets, Logic and Reasoning about Knowledge, vol. 15 of Applied Logic Series, p. 395-417, Kluwer, 1999.

[BLO 00] BLOCH I., LANG J., "Towards mathematical morpho-logics", Proceedings of the Eigth International Conference on Information Processing and Management of Uncertainty in Knowledge-Based Systems (IPMU'00), 2000, p. 1405-1412.

[BLO 04] Bloch I., Pino Pérez R., UzCÁtegui C., “A Unified Treatment for Knowledge Dynamics", Proceedings of the Ninth Conference on Principles of Knowledge Representation and Reasoning (KR'04), 2004, p. 329-337.

[BOO 01] Воотн R., "A negociation-style framework for non-prioritised revision", Proceedings of the Eighth Conference on Theoretical Aspects of Rationality and Knowledge (TARK'01), 2001, p. 137-150.

[BOO 02] Bоотн R., "Social contraction and belief negociation", Proceedings of the Eighth Conference on Principles of Knowledge Representation and Reasoning (KR'02), 2002, p. 374-384.

[CHO 93] CHOLvy L., "Proving theorems in a multi-sources environment", Proceedings of the Thirteenth International Joint Conference on Artificial Intelligence (IJCAI'93), 1993, p. 66-71.

[CHO 95] CHOLVy L., "Automated reasoning with merged contradictory information whose reliability depends on topics", Proceedings of the Third European Conference on Symbolic and Quantitative Approaches to Reasoning and Uncertainty (ECSQARU'95), 1995.

[CHO 98] CHOLvy L., "Reasoning about data provided by federated deductive databases", Journal of Intelligent Information Systems, vol. 10, num. 1, 1998.

[DAL 88] DALAL M., "Investigations into a theory of knowledge base revision: preliminary report", Proceedings of the Seventh American National Conference on Artificial Intelligence (AAAI'88), 1988, p. 475-479.

[EVE 04] EVeraere P., Konieczny S., Marquis P., "On merging strategy-proofness", Proceedings of the Ninth Conference on Principles of Knowledge Representation and Reasoning (KR'04), 2004, p. 357-358.

[GÄR 88] GÄRdENFOrs P., Knowledge in flux, MIT Press, 1988.

[GÄR 92] GÄRdenfors P., Ed., Belief Revision, Cambridge University Press, 1992.

[KAT 91] Katsuno H., Mendelzon A. O., "Propositional knowledge base revision and minimal change", Artificial Intelligence, vol. 52, 1991, p. 263-294. 
294 JANCL - 14/2004. Uncertainty in multiple data sources

[KON 98] Konieczny S., PINO PÉREZ R., "On the logic of merging”, Proceedings of the Sixth International Conference on Principles of Knowledge Representation and Reasoning (KR'98), 1998, p. 488-498.

[KON 99] Konieczny S., Pino PÉRez R., "Merging with Integrity Constraints", Proceedings of the Fifth European Conference on Symbolic and Quantitative Approaches to Reasoning with Uncertainty (ECSQARU'99), LNAI 1638, 1999, p. 233-244.

[KON 00] KONIECZNY S., "On the difference between merging knowledge bases and combining them", Proceedings of the Seventh International Conference on Principles of Knowledge Representation and Reasoning (KR'00), 2000, p. 135-144.

[KON 02a] KONIECZNY S., PINO PÉREZ R., "Merging information under constraints: a qualitative framework", Journal of Logic and Computation, vol. 12, num. 5, 2002, p. 773-808.

[KON 02b] KONIECZny S., PINo PÉREZ R., "On the Frontier between Arbitration and Majority”, Proceedings of the Eighth International Conference on Principles of Knowledge Representation and Reasoning (KR'02), 2002, p. 109-118.

[KON 04] Konieczny S., LAng J., Marquis P., "DA² Merging Operators", Artificial Intelligence, vol. 157, num. 1-2, 2004, p. 49-79.

[LiB 98] Liberatore P., Schaerf M., “Arbitration (or how to merge knowledge bases)", IEEE Transactions on Knowledge and Data Engineering, vol. 10, num. 1, 1998, p. 76-90.

[LIN 99] Lin J., Mendelzon A. O., "Knowledge base merging by majority", Dynamic Worlds: From the Frame Problem to Knowledge Management, Kluwer, 1999.

[REV 93] REVESZ P. Z., "On the semantics of theory change: arbitration between old and new information", Proceedings of the Twelfth ACM SIGACT-SIGMOD-SIGART Symposium on Principles of Databases, 1993, p. 71-92.

[REV 97] REVESZ P. Z., "On the semantics of arbitration", International Journal of Algebra and Computation, vol. 7, num. 2, 1997, p. 133-160.

[SPO 88] SPOHN W., "Ordinal conditional functions: a dynamic theory of epistemic states", Harper W. L., Skyrms B., Eds., Causation in Decision, Belief Change, and Statistics, vol. 2, p. 105-134, Kluwer, 1988. 